\title{
Billiards and the five distance theorem
}

\author{
by \\ JAN FLOREK (Wrocław)
}

1. Introduction. For a real number $x,\{x\}$ is the fractional part of $x$, and $\|x\|$ is the distance between $x$ and the nearest integer.

Let us fix $\theta \in(0,1)$ and $n \in \mathbb{N}$. If $\{m \theta\}$ are inserted into the unit circle (i.e. the circle of unit circumference) for $0 \leq m<n$, the resulting elementary intervals have at most three lengths, one being the sum of the other two. This property, known as the Steinhaus conjecture or the three distance theorem was first proved by Sós [So1], [So2] and then by Surányi [Su] and Świerczkowski $[\mathrm{Sw}]$ (see also [AB] for a recent survey). Later on Geelen and Simpson [GS] proved the five distance theorem: there are at most five lengths when the unit circle is partitioned by the points $\{m \theta\}$ and $\{m \theta+\alpha\}$, for $0 \leq m<n$ (see also $[\mathrm{CG}]$ and $[\mathrm{L}]$ for a " $3 d$-distance" generalization).

A $\theta$-billiard sequence is a sequence $F(j) \in[0,1), j \in \mathbb{N}$, which satisfies the following conditions (see $[\mathrm{F}]$ ):

$$
\begin{array}{ll}
F(j)+F(j+1)=\theta \text { or } 1+\theta & \text { for } j \text { odd, } \\
F(j)+F(j+1)=0 \text { or } 1 & \text { for } j \text { even. }
\end{array}
$$

We consider a billiard table rectangle with perimeter of length 1 with the left top vertex labeled $v_{0}$, and the others, in the clockwise direction, $v_{1}, v_{2}$ and $v_{3}$. The distance from $v_{0}$ to $v_{1}$ is $\theta / 2$. We describe the position of points on the perimeter by their distance along the perimeter measured in the clockwise direction from $v_{0}$, so that $v_{1}$ is at position $\theta / 2, v_{2}$ at $1 / 2$ and $v_{3}$ at $(\theta+1) / 2$. If a billiard ball is pushed from position $F(1)$ at the angle of $\pi / 4$, then it will rebound against the sides of the rectangle consecutively at points $F(2)$, $F(3), \ldots$

Let $n \geq 5$ and $\Phi=\{F(j): 1 \leq j \leq n\}$ be a set of different points. An open connected subset of the perimeter of the billiard rectangle with different endpoints in $\Phi$ is called a segment. A segment with endpoints $F(k)$, $F(l), 1 \leq k, l \leq n$, is called even (or odd), and has weight $|k-l|$ (or

2010 Mathematics Subject Classification: 11J71, 11Z05, 11J04.

Key words and phrases: five distance theorem, billiards. 
$k+l$ ), if $k, l$ are of the same (or different) parity. The length of a segment is a distance along the perimeter between its endpoints. A segment of length less than $1 / 2$ is called short. A short segment with endpoints $F(k), F(k+1), 1 \leq k<n$, is called a corner segment. A segment is called elementary if there are no points of $\Phi$ between its endpoints. Suppose $\emptyset \neq V \subseteq\{F(1), F(n)\}$. A segment $I$ is associated with $V$ if $I$ is an elementary segment incident with an element of $V$ or $I \cap \Phi$ is a nonempty set contained in $V$. Since $n \geq 5$, any segment associated with $\{F(1), F(n)\}$ is uniquely determined by its endpoints. By $(F(k), F(l))$ (respectively $(F(k), F(l))_{\mathrm{e}}$ or $(F(k), F(l))_{\text {a }}$ ) we denote the short segment (respectively the elementary segment or the segment associated with the set $\{F(1), F(n)\})$ incident with $F(k)$ and $F(l)$. Note that $(F(k), F(l))$ and $(F(l), F(k))$ are the same segment.

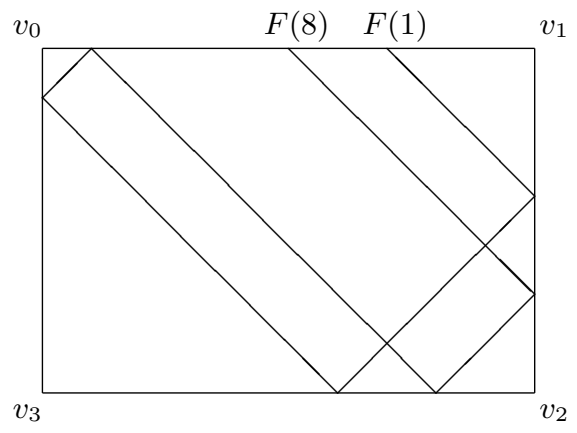

Fig. 1. A chain of odd elementary segments with weight 9: $(F(1), F(8)),(F(2), F(7))$, $(F(3), F(6)),(F(4), F(5))$

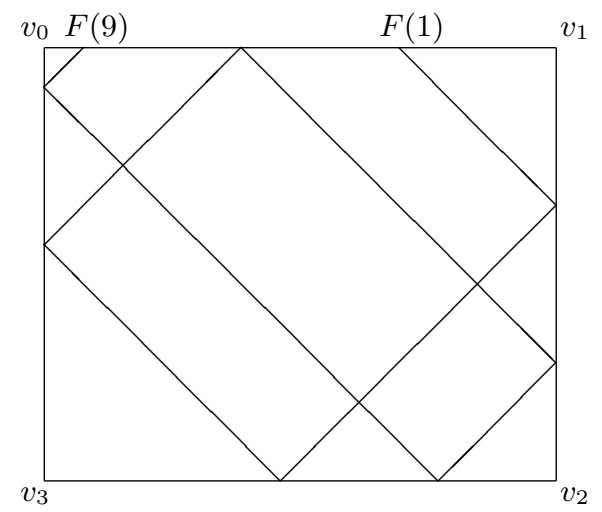

Fig. 2. A chain of even elementary segments with weight $4:(F(1), F(5)),(F(2), F(6))$, $(F(3), F(7)),(F(4), F(8)),(F(5), F(9))$ 
In Lemma 2.3 we prove that short and elementary segments come in equal weight chains with pairs

$$
\ldots,(F(i), F(j)),(F(i+1), F(j-1)),(F(i+2), F(j-2)), \ldots
$$

if $i$ and $j$ have opposite parity (see Fig. 1), and with pairs

$$
\ldots,(F(i), F(j)),(F(i+1), F(j+1)),(F(i+2), F(j+2)), \ldots
$$

if $i$ and $j$ have the same parity (see Fig. 2). Such a chain can end with a corner segment (four possibilities), or with one of the indices $i, j$ equal to 1 (two possibilities), or with one of the indices $i, j$ equal to $n$ (two possibilities), or with the chain being stopped by $F(1)$ or $F(n)$ falling inside what would be the next short and elementary segment. This gives at most ten possible end pairs of the chains, so at most five chains and at most five weights.

Let $\omega_{1}<\omega_{2}$ be odd weights and $\omega_{0}$ be an even weight of segments associated with $\{F(1)\}$, and let $\omega_{3}<\omega_{4}$ be other odd weights of segments associated with $\{F(1), F(n)\}$ (see Corollary 2.2). Suppose $a_{0}, \ldots, a_{4}$ are the lengths of these segments with weights $\omega_{0}, \ldots, \omega_{4}$, respectively. The main result of this paper is Theorem 2.3, which says that the weights of elementary segments have at most five different values $\omega_{0}, \ldots, \omega_{4}$. Elementary segments with equal weights have equal lengths. Moreover, if $A_{i}$ is the set of all elementary segments with weight $\omega_{i}$, then

$$
\left|A_{2}\right|+\epsilon\left|A_{1}\right|=\left|A_{3}\right|+\delta\left|A_{4}\right|=\frac{1}{2} \omega_{0}
$$

and

$$
a_{2}-\epsilon a_{1}=a_{3}-\delta a_{4}=a_{0}
$$

for some $\epsilon, \delta \in\{-1,1\}$. The set $A_{0}$ is a union of at most $\omega_{0}$ sequences, each of them consisting of successively adjacent elementary segments, and the difference between the lengths of two such sequences is at most 1 .

In $[\mathrm{F}]$, it has been proved that a sequence $F(j) \in[0,1), j \in \mathbb{N}$, is a $\theta$-billiard sequence if and only if it satisfies the following conditions:

$$
\begin{aligned}
F(2 j) & =\{j \theta-F(1)\} & & \text { for } j \in \mathbb{N}, \\
F(2 j+1) & =\{-j \theta+F(1)\} & & \text { for } j \in \mathbb{N} \cup\{0\} .
\end{aligned}
$$

This implies that the set $\{F(j): 1 \leq j \leq 2 n\}$ is a union of two sets of points placed consecutively along the unit circle at the constant arc distance $\theta$ in two opposite directions:

(*) $\{F(j): 1 \leq j \leq 2 n\}=\{\{j \theta-\beta\}: 1 \leq j \leq n\} \cup\{\{(n-j) \theta+\gamma\}: 0 \leq j<n\}$, where $\beta=F(1)$ and $\gamma=F(1)-n \theta$.

Let us consider the unit directed circle partitioned into $n$ intervals (called elementary intervals) by the set of $2 n$ different points of two kinds: $\{m \theta\}$ and $\{m \theta+\alpha\}$, where $0 \leq m<n$. For an elementary interval with initial point $\{k \theta\}$ or $\{k \theta+\alpha\}$ and terminal point $\{l \theta\}$ or $\{l \theta+\alpha\}$, we define its 
weight as $l-k$. An elementary interval is said to be even if its endpoints are of the same kind. Other elementary intervals are called odd. We say that two odd intervals are equivalent if they have the same weight and their initial points are of the same kind. Using Lemma 2.3 and $(*)$ one can formulate Theorem 2.3 in the following way: Any two even intervals have the same weight and the same length, say $\omega$ and $a_{0}$. The set of all even intervals is a union of at most $2|\omega|$ sequences, each of them consisting of successively adjacent elementary intervals, and the difference between the lengths of two such sequences is at most 1 . The set of all odd intervals is a union of at most four equivalence classes $A_{1}, A_{2}, A_{3}, A_{4}$, each of them consisting of elementary intervals with equal length, respectively $a_{1}, a_{2}, a_{3}, a_{4}$. Moreover, the following conditions are satisfied:

$$
\left|A_{2}\right|+\epsilon\left|A_{1}\right|=\left|A_{3}\right|+\delta\left|A_{4}\right|=|\omega|
$$

and

$$
a_{2}-\epsilon a_{1}=a_{3}-\delta a_{4}=a_{0}
$$

for some $\epsilon, \delta \in\{-1,1\}$.

2. Five weight theorem. Suppose $0<\theta<1$ and $F(j)$ is a $\theta$-billiard sequence. Let $n \in \mathbb{N}, n \geq 5$. We assume that the elements $F(1), \ldots, F(n)$ are different.

For real numbers $x, y$ we write $x \equiv y$ iff $x \equiv y(\bmod 1)$. Thus we have

$$
\|x\|=\|y\| \quad \text { iff } \quad x \equiv y \text { or } x \equiv-y .
$$

REMARK 2.1. By definition of a $\theta$-billiard sequence we obtain:

$$
F(l)-F(k) \equiv \begin{cases}F(k+1)-F(l+1) & \text { for } k<l \text { of the same parity, } \\ F(k+1)-F(l-1) & \text { for } k<l \text { of different parity }\end{cases}
$$

THEOREM 2.1. Short segments with equal weights have equal lengths.

Proof. Notice that a short segment $(F(k), F(l))$ has length $\|F(k)-F(l)\|$. By Remark 2.1 we have

$$
F(l)-F(k) \equiv(-1)^{n-l}(F(n)-F(k+n-l))
$$

for $k<l$ of the same parity, and

$$
F(l)-F(k) \equiv(-1)^{(l-k-1) / 2}\left(F\left(\frac{l+k+1}{2}\right)-F\left(\frac{l+k-1}{2}\right)\right)
$$

for $k<l$ of different parity. Hence, each short and even (respectively odd) segment $I$ has the same length as the only short segment incident with $F(n)$ (the only corner segment, respectively) which has the same weight as $I$. 
Lemma 2.1. If $(F(k), F(l))$ is a short segment and $F(m) \in(F(k), F(l))$, $1 \leq m \leq n$, then

$$
F\left(m+(-1)^{m}\right) \in\left(F\left(k+(-1)^{k}\right), F\left(l+(-1)^{l}\right)\right)
$$

for $1 \leq m+(-1)^{m}, k+(-1)^{k}, l+(-1)^{l} \leq n$, and

$$
F\left(m-(-1)^{m}\right) \in\left(F\left(k-(-1)^{k}\right), F\left(l-(-1)^{l}\right)\right)
$$

for $1 \leq m-(-1)^{m}, k-(-1)^{k}, l-(-1)^{l} \leq n$.

Proof. Since the segment $(F(k), F(l))$ is short,

$$
\begin{aligned}
(F(k), F(l))=\{x \in[0,1):\|F(k)-x\|+\|x-F(l)\| & =\|F(k)-F(l)\|, \\
x & \neq F(k), x \neq F(l)\} .
\end{aligned}
$$

If $F(m) \in(F(k), F(l))$, then by Theorem 2.1 we obtain

$$
\begin{aligned}
\| F\left(k \pm(-1)^{k}\right)- & F\left(m \pm(-1)^{m}\right)\|+\| F\left(m \pm(-1)^{m}\right)-F\left(l \pm(-1)^{l}\right) \| \\
& =\|F(k)-F(m)\|+\|F(m)-F(l)\| \\
& =\|F(k)-F(l)\|=\left\|F\left(k \pm(-1)^{k}\right)-F\left(l \pm(-1)^{l}\right)\right\|,
\end{aligned}
$$

which ends the proof.

Lemma 2.2. Let the short segment $(F(k), F(l)), k<l$, be elementary. Then the following implications hold:

(1) If $k, l$ are of different parity, then the short segment $(F(k+1)$, $F(l-1))$ is elementary.

(2) If $k, l$ are of the same parity and $l<n(1<k)$, then the short segment $(F(k+1), F(l+1))((F(k-1), F(l-1))$, respectively $)$ is elementary or associated with $\{F(1), F(n)\}$.

(3) If $k, l$ are of different parity and $1<k, l<n$, then the short segment $(F(k-1), F(l+1))$ is elementary or associated with $\{F(1), F(n)\}$.

Proof. (1) Let $F(m) \in(F(k+1), F(l-1))$ for some $1 \leq m \leq n$. If $m$, $k+1$ are of the same parity, then by Lemma 2.1 we have

$$
F(m-1) \in(F(k), F(l)) \quad \text { for } m>1 .
$$

If $m=1$, then by Lemma 2.1,

$$
F(i) \in(F(k+i), F(l-i)) \quad \text { for } 1 \leq i \leq l-k .
$$

Hence $F(l-k) \in(F(l), F(k))$.

If $m, k+1$ are of different parity, then by Lemma 2.1 we have

$$
F(m+1) \in(F(k), F(l)) \quad \text { for } m<n .
$$

If $m=n$ then by Lemma 2.1,

$$
F(n+1-i) \in(F(k+i), F(l-i)) \quad \text { for } 1 \leq i \leq l-k .
$$

Hence $F(n+1-l+k) \in(F(l), F(k))$. 
(2) Let $F(m) \in(F(k+1), F(l+1))$ for some $1<m<n$. If $m, k+1$ are of different parity, then by Lemma 2.1 ,

$$
F(m+1) \in(F(k), F(l)) .
$$

If $m, k+1$ are of the same parity, then by Lemma 2.1 ,

$$
F(m-1) \in(F(k), F(l)) .
$$

(3) Let $F(m) \in(F(k-1), F(l+1))$ for some $1<m<n$. If $m, k-1$ are of different parity, then by Lemma 2.1 ,

$$
F(m-1) \in(F(k), F(l)) .
$$

If $m, k-1$ are of the same parity, then by Lemma 2.1 ,

$$
F(m+1) \in(F(k), F(l)),
$$

which completes the proof.

REMARK 2.2. An odd (respectively even) elementary segment which is not short is of the form $(F(1), F(2))_{\mathrm{e}}$ or $(F(n-1), F(n))_{\mathrm{e}}$ (respectively of the form $\left.(F(1), F(n))_{\mathrm{e}}\right)$.

Theorem 2.2. If $(F(k), F(1))_{\mathrm{e}},(F(1), F(n))_{\mathrm{e}}, 1<k<n$, are elementary segments incident with $F(1)$, then $k$ is even.

Proof. Suppose a contrario that $k$ is odd. Since $k<n$, by Remark 2.2 the elementary segment $(F(k), F(1))_{\mathrm{e}}$ is short. Hence by Lemma $2.2(2)$ there exists $0<j \leq n-k$ such that the short segment $(F(k+j), F(1+j))$ is associated with $\{F(1), F(n)\}$. First we prove

(i) $F(1), F(n) \notin(F(k+j), F(1+j))$.

If $F(n) \in(F(k+j), F(1+j))$, then $F(1) \in(F(k+j), F(1+j))$, because $(F(1), F(n))_{\mathrm{e}}$ is elementary and $F(1) \neq F(k+j), F(1) \neq F(1+j)$. If $F(1) \in(F(k+j), F(1+j))$, then $\|F(k+j)-F(1+j)\|>\|F(k)-F(1)\|$, because $(F(k), F(1))$ is elementary. Hence, by Theorem 2.1 we obtain a contradiction $\|F(k+j)-F(1+j)\|=\|F(k)-F(1)\|$.

Since $(F(k+j), F(1+j))$ is associated with $\{F(1), F(n)\}$, by (i) we have $k+j=n$ and

(ii) the segment $(F(n), F(1+n-k))$ is elementary.

It follows that the segments $(F(k), F(1)),(F(1), F(n))_{\mathrm{e}},(F(n), F(1+n-k))$ are elementary and different. By Theorem 2.1,

$$
F(k)-F(1) \equiv F(n)-F(1+n-k) .
$$

Since $k$ is odd, by Remark 2.1,

$$
F(k)-F(1) \equiv(-1)^{n-k}(F(n)-F(1+n-k)) .
$$


Thus $(-1)^{n-k}=1$ and so

(iii) $n$ is odd.

Since there exists an odd elementary segment which is short, by Lemma 2.2(3) there exists an odd segment associated with $\{F(1), F(n)\}$. Hence, we obtain a contradiction because by (ii) and (iii) all segments associated with $\{F(1), F(n)\}$ are even.

Corollary 2.1. If $(F(1), F(n))_{\mathrm{e}},(F(n), F(l))_{\mathrm{e}}, 1<l<n$, are elementary segments incident with $F(n)$, then $l, n$ are of different parity.

Proof. Set $F^{\prime}(j)=F(n-j+1)$ for $1 \leq j \leq n$. Since $\left(F^{\prime}(n-l+1), F^{\prime}(1)\right)_{\mathrm{e}}$, $\left(F^{\prime}(1), F^{\prime}(n)\right)_{\mathrm{e}}$ are elementary segments incident with $F^{\prime}(1)$, by Theorem 2.2, $n-l+1$ is even.

LEMMA 2.3.

(1) Let $k, l$ be of different parity and suppose $k+1<l$. A short segment $(F(k), F(l))$ is elementary or associated with $\{F(1), F(n)\}$ if and only if the short segment $(F(k+1), F(l-1))$ is elementary.

(2) Let $k, l$ be of the same parity and suppose $k<l<n$. A short segment $(F(k), F(l))$ is elementary or associated with $\{F(1)\}$ if and only if the short segment $(F(k+1), F(l+1))$ is elementary or associated with $\{F(n)\}$.

Proof. (1) Assume that $F(m) \notin(F(k), F(l))$ for $1<m<n$. If $F(2) \in$ $(F(k+1), F(l-1))$ and $k+1$ is even, then by Lemma $2.1, F(1) \in(F(k), F(l))$. Hence we obtain a contradiction since by Theorem $2.2, k$ is even. If $F(n-1)$ $\in(F(k+1), F(l-1))$ and $n-1, l-1$ are of the same parity, then by Lemma 2.1, $F(n) \in(F(k), F(l))$. Hence we obtain a contradiction because by Corollary 2.1, $n, l$ are of different parity. Thus, by the proof of Lemma 2.2(1) (as $l-k>1)$, the segment $(F(k+1), F(l-1))$ is elementary.

The opposite implication follows from Lemma 2.2(3).

(2) Assume that $F(m) \notin(F(k), F(l))$ for $1<m \leq n$. If $F(2) \in(F(k+1)$, $F(l+1))$ and $k+1$ is even, then by Lemma 2.1, $F(1) \in(F(k), F(l))$. Hence we obtain a contradiction because by Theorem $2.2, k$ is even. Thus, by the proof of Lemma 2.2(2), we have

$$
F(m) \notin(F(k+1), F(l+1)) \quad \text { for } 1<m<n .
$$

Hence, if $k+1$ is odd, then by Theorem 2.2, $F(1) \notin(F(k+1), F(l+1))$. If $k+1$ is even and $F(1) \in(F(k+1), F(l+1))$, then by Lemma 2.1 we obtain a contradiction $F(2) \in(F(k), F(l))$.

The proof of the opposite implication is analogous. 
COROLlary 2.2. There exist exactly two odd segments and one even segment associated with $\{F(1)\}$, and exactly four odd segments associated with $\{F(1), F(n)\}$. The weights of these four odd segments are different.

Proof. The first part of Corollary 2.2 follows from Theorem 2.2 and Corollary 2.1. By Lemma 2.3(1) short and odd segments which are associated with $\{F(1), F(n)\}$ have different weights. Suppose $I$ is an odd segment associated with $\{F(1), F(n)\}$ which is not short. Since $n \geq 5$, by Remark 2.2 there are four possibilities for the segment $I$ :

$$
\begin{gathered}
I=(F(1), F(2))_{\mathrm{a}}, \quad I=(F(n-1), F(n))_{\mathrm{a}}, \\
I=(F(2), F(3))_{\mathrm{a}}, \quad F(1) \in I \quad \text { and } \quad F(4) \notin I, \\
I=(F(n-2), F(n-1))_{\mathrm{a}}, \quad F(n) \in I \quad \text { and } \quad F(n-3) \notin I .
\end{gathered}
$$

Hence the weights of $I$ and of any other odd segment associated with $\{F(1)$, $F(n)\}$ are different.

TheOREM 2.3. Let $\omega_{1}<\omega_{2}$ be odd weights and $\omega_{0}$ be an even weight of segments associated with $\{F(1)\}$, and let $\omega_{3}<\omega_{4}$ be other odd weights of segments associated with $\{F(1), F(n)\}$. Let $a_{0}, \ldots, a_{4}$ be the lengths of those segments with weights $\omega_{0}, \ldots, \omega_{4}$, respectively.

(1) The weights of elementary segments have at most five different values $\omega_{0}, \ldots, \omega_{4}$. Moreover,

$$
\omega_{2}-\omega_{1}=\omega_{4}-\omega_{3}=\omega_{0} .
$$

If there exists an elementary segment $(F(1), F(n))_{\mathrm{e}}$, then any other elementary segment is odd.

(2) Elementary segments with equal weights have equal lengths.

(3) If $A_{i}$ is the set of all elementary segments with weight $\omega_{i}, 0 \leq i \leq 4$, then

$$
\left|A_{2}\right|+\epsilon\left|A_{1}\right|=\left|A_{3}\right|+\delta\left|A_{4}\right|=\frac{1}{2} \omega_{0}
$$

and

$$
a_{2}-\epsilon a_{1}=a_{3}-\delta a_{4}=a_{0}
$$

for some $\epsilon, \delta \in\{-1,1\}$.

(4) The set $A_{0}$ is a union of at most $\omega_{0}$ sequences, each of them consisting of successively adjacent elementary segments, and the difference between the lengths of two such sequences is at most 1.

Proof. (1) By Corollary 2.2 the weights $\omega_{0}, \ldots, \omega_{4}$ are different and well defined. By Lemma 2.3 and Remark 2.2 the weights of elementary segments have at most five different values $\omega_{0}, \ldots, \omega_{4}$. Suppose that there exists an elementary segment $(F(1), F(n))_{\mathrm{e}}$. If there exists an even elementary segment, not equal to $(F(1), F(n))_{\mathrm{e}}$, then by Remark 2.2 it is short, and by 
Lemma 2.3(2) there exists an even elementary segment $(F(k), F(1))_{\mathrm{e}}$ for some $k<n$. However, this is impossible by Theorem 2.2.

(2) By Theorem 2.1 short and elementary segments with equal weights have equal lengths. Suppose $I$ is an elementary segment which is not short. If $I$ is even, then by Remark 2.2 it is of the form $(F(1), F(n))_{\mathrm{e}}$. Hence by (1) any other elementary segment is odd. If $I$ is odd, then by Remark 2.2 the weights of $I$ and of any other elementary segment are different.

(3) Suppose $(F(k), F(1))_{\mathrm{e}},(F(1), F(l))_{\mathrm{e}}, k<l$, are elementary segments incident with $F(1)$, and $(F(r), F(n))_{\mathrm{e}},(F(n), F(t))_{\mathrm{e}}, r<t$, are elementary segments incident with $F(n)$. Let us consider the following cases:

(i) $k, l$ are of different parity,

(ii) $k, l$ are of the same parity,

(iii) $r, t$ are of different parity,

(iv) $r>1$ and $r, t$ are of the same parity,

(v) $r=1$ and $t$ is odd.

(i) By Theorem $2.2 k$ is even and $l$ is odd. Hence

$$
\omega_{2}-\omega_{1}=(l+k)-(k+1)=\omega_{0}, \quad a_{2}-a_{1}=a_{0} .
$$

If $(F(k), F(l))_{\mathrm{a}}$ is short, then by Lemma 2.3(1),

$$
\begin{aligned}
& A_{1}=\left\{(F(1+i), F(k-i)): 0 \leq i \leq \frac{k}{2}-1\right\}, \\
& A_{2}=\left\{(F(k+i), F(l-i)): 0<i \leq \frac{l-k-1}{2}\right\} .
\end{aligned}
$$

Hence

$$
\left|A_{2}\right|+\left|A_{1}\right|=\frac{l-k-1}{2}+\frac{k}{2}=\frac{\omega_{0}}{2} .
$$

If $(F(k), F(l))_{\mathrm{a}}$ is not short, then by Remark 2.2 and Lemma 2.3(1),

$$
l=k+1, \quad\left|A_{2}\right|=0 \quad \text { and } \quad\left|A_{1}\right|=\frac{k}{2} .
$$

(ii) By Theorem $2.2 k$ and $l$ are even. Hence

$$
\omega_{2}-\omega_{1}=(l+1)-(k+1)=\omega_{0}, \quad a_{2}+a_{1}=a_{0} .
$$

Since $l>2$, by Remark $2.2(F(1), F(l))_{\mathrm{e}}$ is short. If $(F(k), F(1))_{\mathrm{e}}$ is short, then by Lemma 2.3(1),

$$
\left|A_{2}\right|-\left|A_{1}\right|=\frac{l}{2}-\frac{k}{2}=\frac{\omega_{0}}{2} .
$$

If $(F(k), F(1))_{\mathrm{e}}$ is not short, then by Remark 2.2 and Lemma 2.3(1),

$$
k=2 \text { and }\left|A_{1}\right|=1 .
$$

(iii) By Corollary 2.1, $t, n$ are of different parity and $r, n$ are of the same parity. If $r=1$, then $n=l$ and $n-r=l-1=\omega_{0}$. If $r \neq 1$, then by Remark 
2.2 the even segment $(F(r), F(n))_{\mathrm{e}}$ is short and by $(1), n-r=\omega_{0}$. Hence

$$
\omega_{4}-\omega_{3}=(n+t)-(t+r)=\omega_{0}, \quad a_{3}-a_{4}=a_{0} .
$$

If $(F(r), F(t))_{\mathrm{a}}$ is short, then by Lemma 2.3(1),

$$
\left|A_{3}\right|+\left|A_{4}\right|=\frac{t-r-1}{2}+\frac{n-t+1}{2}=\frac{\omega_{0}}{2} .
$$

If $(F(r), F(t))_{\mathrm{a}}$ is not short, then by Remark 2.2 and Lemma 2.3(1),

$$
t=r+1, \quad\left|A_{3}\right|=0 \quad \text { and } \quad\left|A_{4}\right|=\frac{n-t+1}{2} .
$$

(iv) By Corollary 2.1, $t, n$ are of different parity and $r, n$ are of different parity. Since $r>1$, by Remark 2.2 the even segment $(F(r), F(t))_{\mathrm{a}}$ is short. Hence by Lemma 2.3(2),

$$
\omega_{4}-\omega_{3}=(n+t)-(n+r)=\omega_{0}, \quad a_{3}+a_{4}=a_{0} .
$$

Since $(F(r), F(n))_{\mathrm{e}}$ and $(F(n), F(t))_{\mathrm{e}}$ are short, by Lemma 2.3(1),

$$
\left|A_{3}\right|-\left|A_{4}\right|=\frac{n-r+1}{2}-\frac{n-t+1}{2}=\frac{\omega_{0}}{2} .
$$

(v) By Theorem 2.2 and Corollary 2.1, $k, n=l$ are even and $k<t$. Hence

$$
\omega_{4}-\omega_{3}=(n+t)-(t+k)=\omega_{0}, \quad a_{3}-a_{4}=a_{0} .
$$

If $(F(k), F(t))_{\mathrm{a}}$ is short, then by Lemma 2.3(1),

$$
\left|A_{3}\right|+\left|A_{4}\right|=\frac{t-k-1}{2}+\frac{n-t+1}{2}=\frac{\omega_{0}}{2} .
$$

If $(F(k), F(t))_{\mathrm{a}}$ is not short, then by Remark 2.2 and Lemma 2.3(1),

$$
t=k+1, \quad\left|A_{3}\right|=0 \quad \text { and } \quad\left|A_{4}\right|=\frac{n-t+1}{2} .
$$

(4) If there exists an elementary segment $(F(1), F(n))_{\mathrm{e}}$, then (4) follows from (1). Let $I=(F(k), F(l))_{\mathrm{a}}$ and $J=(F(k+s), F(l+s))_{\mathrm{a}}, k<l<n$, $s>0$, be even segments associated with $\{F(1)\}$ and $\{F(n)\}$, respectively. By Remark 2.2 the segments $I$ and $J$ are short. Hence by Lemma 2.3(2),

$$
A_{0} \backslash\{I, J\}=\{(F(k+i), F(l+i)): 0<i<s\} .
$$

By Theorem 2.2 and Corollary 2.1: if $(F(x), F(y)),(F(y), F(z)), x<z$, are even elementary segments, then $x<y<z$. Hence we obtain (4).

Acknowledgments. The author is very grateful to an anonymous referee for the very detailed remarks which helped to improve the paper.

\section{References}

[AB] P. Alessandri and V. Berthé, Three distance theorem and combinatorics on words, Enseign. Math. 44 (1998), 103-132. 
[CG] F. R. K. Chung and R. L. Graham, On the set of distances determined by the union of arithmetic progressions, Ars Combin. 1 (1976), 57-76.

[F] J. Florek, Billiard and diophantine approximation, Acta Arith. 134 (2008), 317327.

[GS] J. F. Geelen and R. J. Simpson, A two dimensional Steinhaus theorem, Australas. J. Combin. 8 (1993), 169-197.

[L] F. M. Liang, A short proof of the 3d distance theorem, Discrete Math. 28 (1979), 325-326.

[So1] V. T. Sós, On the theory of diophantine approximations I, Acta Math. Acad. Sci. Hungar. 8 (1957), 461-472.

[So2] - On the distribution mod 1 of the sequence n $\alpha$, Ann. Univ. Sci. Budapest. Eötvös Sect. Math. 1 (1958), 127-134.

[Su] J. Surányi, Über die Anordnung der Vielfachen einer reellen Zahl mod 1, ibid., 107-111.

[Sw] S. Świerczkowski, On successive settings of an arc on the circumference of a circle, Fund. Math. 46 (1958), 187-189.

Institute of Mathematics

University of Economics

Komandorska 118/120

53-345 Wrocław, Poland

E-mail: jan.florek@ue.wroc.pl

Received on 16.7.2008

and in revised form on 3.6.2009 